\title{
Influence of Sr Content on CMR Effect in Polycrystalline $\mathrm{La}_{1-x} \mathrm{Sr}_{x} \mathrm{MnO}_{3}$ Thin Films
}

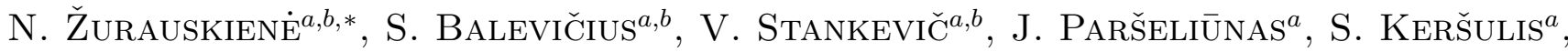 \\ A. Abrutis ${ }^{c}$ And V. Plaušinaitiene $\dot{E}^{a, c}$ \\ ${ }^{a}$ Semiconductor Physics Institute, A. Goštauto 11, Vilnius, Lithuania \\ ${ }^{b}$ Vilnius Gediminas Technical University, Sauletekio 11, Vilnius, Lithuania \\ ${ }^{c}$ Vilnius University, Dep. of Chemistry, Naugarduko 24, Vilnius, Lithuania
}

\begin{abstract}
The magnetoresistance of thin polycrystalline $\mathrm{La}_{1-x} \mathrm{Sr}_{x} \mathrm{MnO}_{3}$ films deposited on lucalox substrate using metal organic chemical vapor deposition technique was investigated in pulsed magnetic fields up to $18 \mathrm{~T}$ in the temperature range 100-320 K. The influence of film preparation conditions, ambient temperature variation and $\mathrm{Sr}$ content is analyzed in order to determine the optimal conditions for the design of CMR- $B$-scalar magnetic field sensor based on thin manganite film, operating at room temperature.
\end{abstract}

PACS numbers: 75.47.Gk, 75.47.Lx, 75.30.Hx, 85.70.Kh

\section{Introduction}

The measurement of high magnetic field (5-50 T) up to now is serious technical task. Usually used inductive, galvanomagnetic or magnetooptical sensors are sensitive to magnetic field direction. Thus for exact measurement of absolute value of magnetic induction the direction of magnetic field has to be known in advance. This is disadvantage in case of dynamic measurements performed during operation of linear electromagnetic motors, electromagnetic launchers and magnetocumulative generators. Therefore, the sensors which could measure magnitude of the magnetic field ( $B$-scalar sensors) independently of its direction are very important for these applications. It was demonstrated that thin manganite films exhibiting collosal magnetoresistance (CMR) phenomenon [1] can be used for measurements of pulsed magnetic field inductance up to $40 \mathrm{~T}$ [2]. The CMR- $B$-scalar sensors were successfully used for highly local measurements of transient magnetic fields during railgun experiments [3]. Therefore, it is of great importance to investigate influence of preparation conditions, ambient temperature variations and composition on magnetoresistance of manganite films used for these sensors design.

In this paper, the results of study of $\mathrm{Sr}$ content influence on magnetoresistance (MR) value, temperature sensitivity and anisotropy (dependence on magnetic field direction relative to film plane) in polycrystalline $\mathrm{La}_{1-x} \mathrm{Sr}_{x} \mathrm{MnO}_{3}$ films are presented.

* corresponding author; e-mail: zurausk@pfi.lt

\section{Experimental}

The $\mathrm{La}_{1-x} \mathrm{Sr}_{x} \mathrm{MnO}_{3}$ (LSMO) films were deposited by metal organic chemical vapor deposition (MOCVD) technique using a vertical hot wall injection CVD reactor [4]. The films were grown on lucalox substrate at $750^{\circ} \mathrm{C}$. The composition (Sr content $x$ ) was changed over the range $0.17-0.3$. Thickness of the films was $400 \mathrm{~nm}$. The polycrystalline structure of the LSMO films was determined from the reflection high-energy electron diffraction (RHEED) measurements. The samples were prepared in a co-planar shape with the $0.5 \times 0.5 \mathrm{~mm}$ square $\mathrm{Ag}$ electrodes deposited by thermal evaporation and separated by a distance of $50 \mu \mathrm{m}$. The electrodes were annealed in $\mathrm{O}_{2}$ atmosphere at $420^{\circ} \mathrm{C}$ for $20 \mathrm{~min}$.

The specific resistance $\rho$ dependence on temperature $T$ was investigated by using a low dc electric field in a closed cycle helium gas cryocooler over the temperature range from $5 \mathrm{~K}$ to $300 \mathrm{~K}$. The MR measurements were performed in a temperature range $100-320 \mathrm{~K}$ using pulsed magnetic field generator [2] equipped with multi-shot magnetic field coil that produced sinus waveform $0.6 \mathrm{~ms}$ duration magnetic pulses with amplitudes up to $18 \mathrm{~T}$.

\section{Results and discussion}

The investigated films were characterized by measuring their resistivity dependence on temperature (see Fig. 1a). The dependences show typical for manganite film metalinsulator transitions at a certain critical temperature $T_{\mathrm{m}}$ (the resistivity maximum $\rho_{\mathrm{m}}$ temperature). The $T_{\mathrm{m}}$ and $\rho_{\mathrm{m}}$ for the films (1)-(3) grown at the same conditions 
were obtained over the temperature range of $235-260 \mathrm{~K}$ and resistivity range of $0.9-3.5 \Omega \mathrm{cm}$, respectively, depending on manganite film composition. An increase of $x$ results in the shift of the $T_{\mathrm{m}}$ to higher temperatures and the decrease of the $\rho_{\mathrm{m}}$. The curve (2f) corresponds to the sample which was prepared at a faster cooling rate of the film after deposition: $40 \mathrm{~min}$ from $750^{\circ} \mathrm{C}$ down to $27^{\circ} \mathrm{C}$ (1-3 curves correspond to cooling for $1.5 \mathrm{~h}$ ). The lower $T_{\mathrm{m}}(215 \mathrm{~K})$ and much higher $\rho_{\mathrm{m}}(12 \Omega \mathrm{cm})$ indicate that this film has more defected structure. The polycrystalline films can be imagined to be a network of tunnel junctions, i.e. high quality crystallites with grain boundaries (GBs) of vitreous structure between them. The total resistivity of the film is mostly determined by the high resistivity of the GBs. The curve (2f) shows that in this case more defects are induced in GBs.

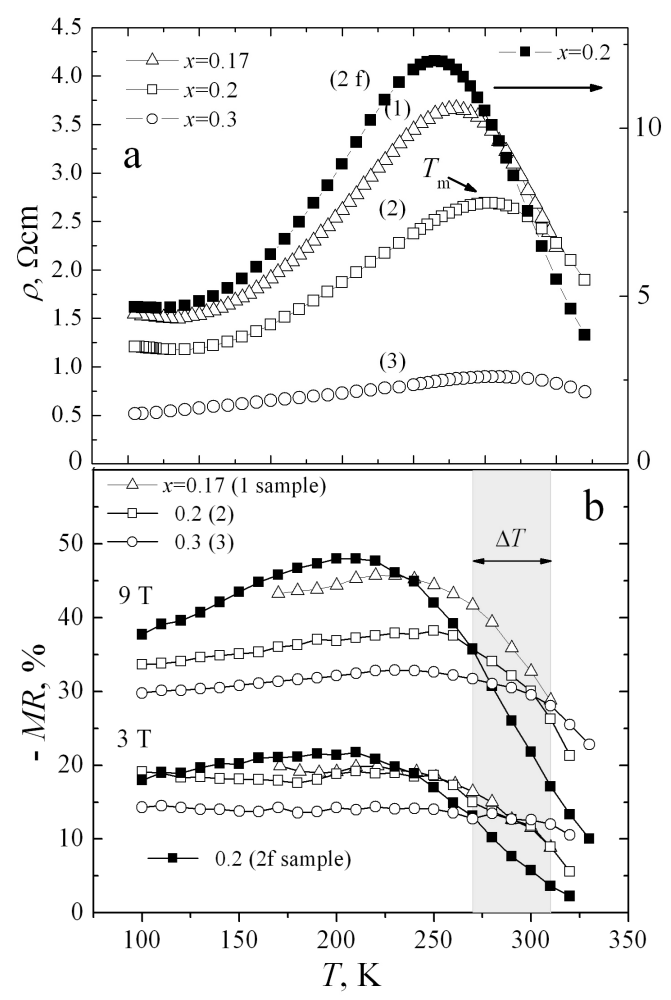

Fig. 1. Dependences of resistivity (a) and negative magnetoresistance (b) on temperature for $\mathrm{La}_{1-x} \mathrm{Sr}_{x} \mathrm{MnO}_{3}$ films with various Sr content $x$.

\subsection{Magnetoresistance in high magnetic fields}

The dependences of negative magnetoresistance on temperature at $3 \mathrm{~T}$ and $9 \mathrm{~T}$ magnetic fields are presented in Fig. 1b. The MR was defined by the formula $\mathrm{MR}=[R(B)-R(0)] / R(0)$, where $R(0)$ and $R(B)$ are film resistances in magnetic field $B$ and without it, respectively. It was found that the MR is large at low temperatures and starts to decrease when $T$ approaches $T_{\mathrm{m}}$ value. The higher is $\rho_{\mathrm{m}}$ and lower $T_{\mathrm{m}}$ of the films (see Fig. 1a), the higher is maximal magnetoresistance $\left(\mathrm{MR}_{\max }\right)$ value obtained in all measured temperature ranges. The decrease of $\mathrm{MR}_{\max }$ with increase of $x$ for polycrystalline films is mostly determined by the ratio between density of $\mathrm{Mn}^{4+}$ and $\mathrm{Mn}^{3+}$ as in the case of epitaxial films [4]. At low temperatures spin polarized tunneling through GBs are expected to be the main reasons of the MR. The figure demonstrates that MR values can be easily tuned by changing the Sr concentration of the films.

In order to determine optimal parameters of the film for design of magnetic field sensor operating at room temperature we will consider in more detail the temperature range $\Delta T=290 \mathrm{~K} \pm 20 \mathrm{~K}$ (see shadow region in Fig. 1b). It is evident that MR values of sample (2f) are significantly lower at this temperature range as compared with samples (1)-(3). At the room temperature which is far from $T_{\mathrm{m}}$ the (2f) film is at pure paramagnetic state which results in small CMR effect.

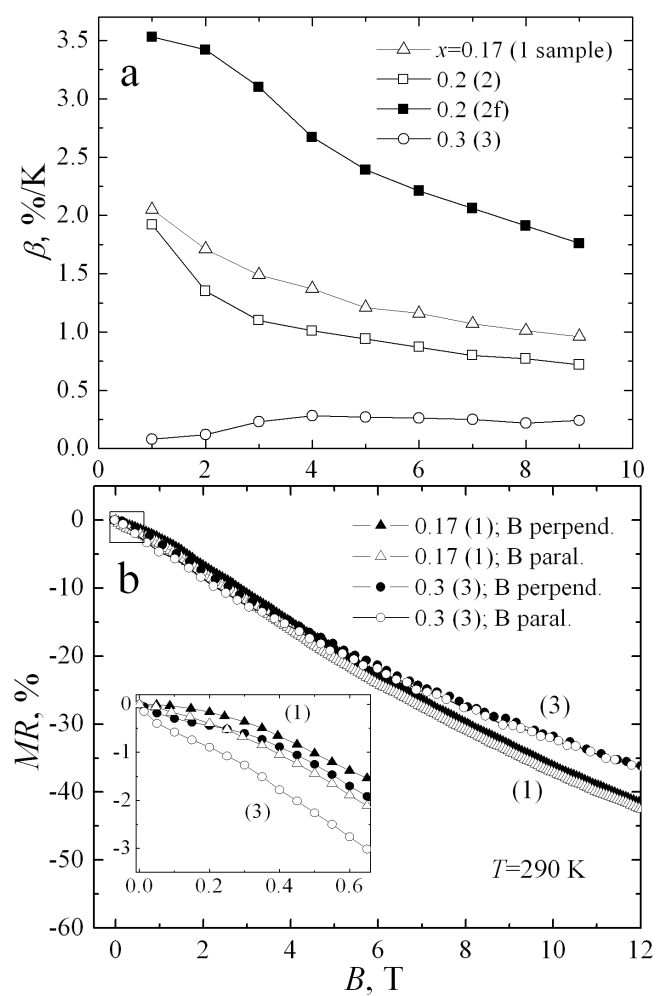

Fig. 2. Temperature sensitivity (a) and magnetoresistance (b) dependences on magnetic induction. Inset shows low field MR.

\subsection{MR temperature sensitivity}

One of important parameters for the device performance is sensitivity to ambient temperature variations. We will use the following coefficient characterizing this sensitivity: $\beta=\left[\Delta \mathrm{MR}_{\max } /\left(\mathrm{MR}_{0 T} \Delta T\right)\right] \times 100 \%$. Here $\Delta \mathrm{MR}_{\text {max }}$ is maximal change of the MR in operation temperature range $\Delta T=T_{2}-T_{1}$ (in our case $T_{2}=310 \mathrm{~K}$, $\left.T_{1}=270 \mathrm{~K}\right), \mathrm{MR}_{0 T}$ is $\mathrm{MR}$ at given magnetic field at central operation temperature $\left.T_{\mathrm{op}}=\left(T_{1}+T_{2}\right) / 2=290 \mathrm{~K}\right)$. 
Figure 2a shows $\beta$ dependence on magnetic inductance value. One can see that for sensor based on (2f) film $\beta$ is too high. For film with $x=0.3$ the sensitivity is the smallest $(\sim 0.2 \% / \mathrm{K})$. This is the result of high $T_{\mathrm{m}}$ which is close to room temperature and, therefore, "flat" enough MR dependence on $T$ around room temperature is observed.

\subsection{Anisotropy of magnetoresistance}

The MR of the films was measured in two magnetic field configurations: when $B$ is parallel $\left(\mathrm{MR}_{\|}\right)$and perpendicular $\left(\mathrm{MR}_{\perp}\right)$ to film plane. The magnetoresistance anisotropy, defined as $\left.\mathrm{MRA}=\left[\mathrm{MR}_{\|}-\mathrm{MR}_{\perp}\right) / \mathrm{MR}_{\|}\right] \times$ $100 \%$, was investigated in low and high magnetic fields. The MR dependences on magnetic field induction for two samples $(x=0.17$ and 0.3$)$ are presented in Fig. 2b. Inset shows the data in low fields $(0-0.65 \mathrm{~T})$. One can see that magnetoresistance anisotropy is higher for film with higher $x$ in low fields: $30 \%$ for (1) and $40 \%$ for (3) in $0.6 \mathrm{~T}$. It has to be mentioned that magnetic field sensor based on CMR film is in general calibrated for average value of the $M R: M R_{a v}$. $=\left(M R_{\|}+M R_{\perp}\right) / 2$. In such a case the measurement error for sensors based on (1) and (3) films is $15 \%$ and $20 \%$, respectively. At high fields the obtained MRA is similar for all investigated samples and does not exceed $3 \%$. Detailed measurements of the MRA showed that for design of $B$-scalar sensor with $B$ measurement error $<10 \%$ in magnetic fields $>1 \mathrm{~T}$, the LSMO film with $x=0.17$ would be preferable.

\section{Conclusions}

It was obtained that in wide temperature range up to room temperature the MR value decreases with in- crease of Sr content $x$. The temperature sensitivity of MR is lowest for films with highest $x$. It was found that for polycrystalline films the value of $\mathrm{MR}$ is sensitive to magnetic field direction at low fields $(<1 \mathrm{~T})$. The magnetoresistance anisotropy is higher for films with high $x$ (high $T_{\mathrm{m}}$ ). In magnetic fields $>3 \mathrm{~T}$, the anisotropy effect was less than $3 \%$. For design of CMR- $B$-scalar sensor operating at room temperature the films with composition $x=0.17$ exhibiting low enough anisotropy effect and showing significant magnetoresistance values $(\approx 36 \%$ at $9 \mathrm{~T})$ are preferable. When $B$-direction is known in advance, the films with $x=0.3$ have advantage due to their smaller sensitivity to temperature variations.

\section{Acknowledgments}

The work was supported by the Lithuanian Science and Studies Foundation contract No. B-21/2008.

\section{References}

[1] C. Israel, M.J. Calderón, N.D. Mathur, Mater. Today 10, 24 (2007).

[2] S. Balevičius, N. Žurauskienè, V. Stankevič, S. Keršulis, J. Novickij, L.L. Altgilbers, F. Clarke, Acta Phys. Pol. A 107, 207 (2005).

[3] M. Schneider, R. Schneider, V. Stankevič, S. Balevičius, N. Žurauskienè, IEEE Trans. Magn. 43, 370 (2007).

[4] A. Abrutis, V. Plausinaitiene, V. Kubilius, A. Teiserskis, Z. Saltyte, R. Butkute, J.P. Senateur, Thin Solid Films 413, 32 (2002). 